\title{
Effects of sevoflurane anesthesia and abdominal surgery on the systemic metabolome: a prospective observational study
}

Yiyong Wei ${ }^{1,2+}$, Donghang Zhang ${ }^{1,2+}$, Jin Liu ${ }^{1,2^{*}}$, Mengchan Ou ${ }^{1,2}$, Peng Liang ${ }^{2}$, Yunxia Zuo ${ }^{2^{*}}$ and Cheng Zhou ${ }^{1}$

\begin{abstract}
Background: Metabolic status can be impacted by general anesthesia and surgery. However, the exact effects of general anesthesia and surgery on systemic metabolome remain unclear, which might contribute to postoperative outcomes.

Methods: Five hundred patients who underwent abdominal surgery were included. General anesthesia was mainly maintained with sevoflurane. The end-tidal sevoflurane concentration ( $E T_{\text {sevo }}$ ) was adjusted to maintain BIS (Bispectral index) value between 40 and 60 . The mean $\mathrm{ET}_{\text {sevo }}$ from $20 \mathrm{~min}$ after endotracheal intubation to $2 \mathrm{~h}$ after the beginning of surgery was calculated for each patient. The patients were further divided into low $\mathrm{ET}_{\text {sevo }}$ group (mean - SD) and high $\mathrm{ET}_{\text {sevo }}$ group (mean + SD) to investigate the possible metabolic changes relevant to the amount of sevoflurane exposure.

Results: The mean $\mathrm{ET}_{\text {sevo }}$ of the 500 patients was $1.60 \% \pm 0.34 \%$. Patients with low $\mathrm{ET}_{\text {sevo }}(n=55)$ and high $\mathrm{ET}_{\text {sevo }}$ $(n=59)$ were selected for metabolomic analysis $(1.06 \% \pm 0.13 \%$ vs. $2.17 \% \pm 0.16 \%, P<0.001)$. Sevoflurane and abdominal surgery disturbed the tricarboxylic acid cycle as identified by increased citrate and cis-aconitate levels and impacted glycometabolism as identified by increased sucrose and D-glucose levels in these 114 patients. Glutamate metabolism was also impacted by sevoflurane and abdominal surgery in all the patients. In the patients with high $\mathrm{ET}_{\text {sevo, }}$ levels of L-glutamine, pyroglutamic acid, sphinganine and L-selenocysteine after sevoflurane anesthesia and abdominal surgery were significantly higher than those of the patients with low $\mathrm{ET}_{\text {sevo, }}$ suggesting that these metabolic changes might be relevant to the amount of sevoflurane exposure.

Conclusions: Sevoflurane anesthesia and abdominal surgery can impact principal metabolic pathways in clinical patients including tricarboxylic acid cycle, glycometabolism and glutamate metabolism. This study may provide a resource data for future studies about metabolism relevant to general anaesthesia and surgeries.
\end{abstract}

Trial registration: www.chictr.org.cn. identifier: ChiCTR1800014327.

Keywords: Metabonomics, Sevoflurane, Observational study, Abdominal surgery

\footnotetext{
*Correspondence: scujinliu@gmail.com; zuoyunxia@scu.edu.cn

${ }^{\dagger}$ Yiyong Wei and Donghang Zhang contributed equally to this work.

'Laboratory of Anesthesia \& Critical Care Medicine, Translational

Neuroscience Center, West China Hospital of Sichuan University, 37\# Guoxue

Xiang, Chengdu 610041, Sichuan, China

2Department of Anesthesiology, West China Hospital of Sichuan University,

37\# Guoxue Xiang, Chengdu 610041, Sichuan, China
}

(c) The Author(s). 2021 Open Access This article is licensed under a Creative Commons Attribution 4.0 International License, which permits use, sharing, adaptation, distribution and reproduction in any medium or format, as long as you give appropriate credit to the original author(s) and the source, provide a link to the Creative Commons licence, and indicate if changes were made. The images or other third party material in this article are included in the article's Creative Commons licence, unless indicated otherwise in a credit line to the material. If material is not included in the article's Creative Commons licence and your intended use is not permitted by statutory regulation or exceeds the permitted use, you will need to obtain permission directly from the copyright holder. To view a copy of this licence, visit http://creativecommons.org/licenses/by/4.0/. The Creative Commons Public Domain Dedication waiver (http://creativecommons.org/publicdomain/zero/1.0/) applies to the data made available in this article, unless otherwise stated in a credit line to the data. 


\section{Introduction}

Although volatile anesthetics have been used in clinical setting for more than 170 years, how volatile anesthetics impact physiological status is still elusive [1]. It is widely known that general anesthetics can impact some important processes of metabolism [2,3] and metabolic connectivity [4]. For example, general anesthesia induced by sevoflurane can change metabolic activity in the central nervous system detected by functional neuroimaging [5]. Sevoflurane inhibits insulin secretion in rodents $[2,6]$ and general anesthesia with sevoflurane is associated with hyperglycaemia in human [7]. However, the exact modulation of general anesthetics on systemic metabolome in human has not been clearly illustrated. Understanding the exact effects of volatile anesthetics on systemic metabolism is important for perioperative management of patients, because it can be involved in postoperative recovery [8], postoperative cognitive function [9] and infection [10].

Metabolic status may impact sensitivity of general anesthetics in turn. Previous study indicates that sensitivity to general anesthetics is associated with metabolic function and/or status of the central nervous system. Impaired metabolic status caused by mitochondrial dysfunction, can alter the effects of volatile anesthetics on neural functions including disrupting excitatory neurotransmitter dynamics [11] and activity of thalamocortical circuit [12]. Isoflurane at clinically relevant concentrations can inhibit mitochondrial complex I, and function of mitochondrial complex I in excitatory neurons is relevant to the sensitivity of volatile anesthetics in vivo in mice [11]. The C. elegans lacking mitochondrial complex I subunit NDUFS4 is remarkably hypersensitive to isoflurane [13]. Oxidative phosphorylation-induced complex I dysfunction can also alter sensitivity to volatile anesthetics [13]. However, whether volatile anesthetics dose-dependently modulate metabolic function in humans is unknown.

In the present study, we hypothesized that general anesthesia and abdominal surgery can impact principal metabolic pathways in humans. Here we conducted a clinical observation to investigate the exact effects of sevoflurane and abdominal surgery on systemic metabolism for the first time.

\section{Methods}

\section{Participants}

The observational study was approved by the Ethical Committee of West China Hospital of Sichuan University on May 19, 2017 (Approval No. 78) and was registered prior to patient enrolment with the Chinese Clinical Trial Registry (ChiCTR1800014327, principal investigator, Yunxia Zuo) on Jan 6, 2018. All selected patients were aged 18-65 years with ASA physical status 1-2, and they underwent abdominal surgery with a duration exceeding
$2 \mathrm{~h}$ between January 2018 and September 2018 at West China Hospital of Sichuan University. Written informed consent was obtained from all patients. The exclusion criteria were as follows: allergic to volatile anesthetics, cerebrovascular diseases, severe cardiovascular diseases, metabolic diseases/disorders, alcohol addiction, malignant hyperthermia, or abnormal liver or renal function. This study adheres to the Strengthening the Reporting of Observational studies in Epidemiology (STROBE) guidelines.

\section{Management of general anesthesia}

No preoperative medication was given. $\mathrm{SPO}_{2}$ (Pulse oxygen saturation), BP (Blood pressure), ECG (Electrocardiograph) and BIS (Bispectral index) were routinely monitored in all the patients. After pre-oxygen $(8 \mathrm{~L} / \mathrm{min}$ oxygen for approximately $3 \mathrm{~min}$ ), the anesthetic circuit was primed with $8 \%$ sevoflurane. Patients inhaled 8\% sevoflurane for anesthesia induction and tracheal intubation was facilitated with sufentanil $\left(0.2-0.4 \mu \mathrm{g} \cdot \mathrm{kg}^{-1}\right)$ and cis-atracurium (0.2-0.3 $\left.\mathrm{mg} \cdot \mathrm{kg}^{-1}\right)$. Then, the lungs were ventilated with $50 \%$ oxygen balanced with air and end-tidal partial pressure of carbon dioxide $\left(\mathrm{P}_{\mathrm{ET}} \mathrm{CO}_{2}\right)$ was maintained between 35 and 45 $\mathrm{mmHg}$. After endotracheal intubation, end-tidal sevoflurane concentration $\left(\mathrm{ET}_{\text {sevo }}\right)$ was adjusted by turning volatile tank concentration up or down $(0.5-1 \%)$ to maintain BIS value at 40-60 while continuous infusion of sufentanil with a rate of $0.1-0.2 \mu \mathrm{g} \cdot \mathrm{kg}^{-1} \cdot \mathrm{h}^{-1}$, adjusted according to vital signs. Patients received bolus of cis-atracurium and additional sufentanil according to clinical requirements during anesthesia maintenance. $\mathrm{ET}_{\text {sevo }}$ was automatically recorded using an anesthetic gas monitor (M1026B; Philips Medizin Systeme, Boblingen, Germany). Sevoflurane was discontinued approximately 10-20 min before the end of surgery. All patients were visited in the post-anesthesia care unit on postoperative day 1 and day 3 to assess intraoperative recall.

\section{Trial grouping}

To investigate dose-dependent effect of sevoflurane on systemic metabolome, the patients were divided into high $\mathrm{ET}_{\text {sevo }}$ and low $\mathrm{ET}_{\text {sevo }}$ groups as following described. The patients in the high $\mathrm{ET}_{\text {sevo }}$ group might receive more sevoflurane than that of the patients in the low $\mathrm{ET}_{\text {sevo }}$ group during the surgery while their BIS values were similar. In total, 500 patients were enrolled. The mean value of $\mathrm{ET}_{\text {sevo }}$ from $20 \mathrm{~min}$ after endotracheal intubation to $2 \mathrm{~h}$ after the beginning of surgery was calculated for each patient, from which population $\mathrm{ET}_{\text {sevo }}$ was yielded. Low $\mathrm{ET}_{\text {sevo }}$ was denoted as $\mathrm{ET}_{\text {sevo }}$ value 1-time $\mathrm{SD}$ lower than population mean $\mathrm{ET}_{\text {sevo. }}$ High $\mathrm{ET}_{\text {sevo }}$ was denoted as $\mathrm{ET}_{\text {sevo }}$ value 1-time $\mathrm{SD}$

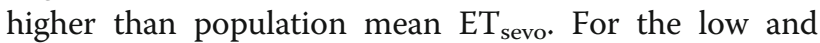
high $\mathrm{ET}_{\text {sevo }}$ groups, we randomly paired 59 patients based on age, BMI (Body mass index), gender, surgical 
procedure (laparotomy or laparoscopy), BIS, vital signs and $\mathrm{P}_{\mathrm{ET}} \mathrm{CO}_{2}$. After pairing, we divided the patients into the low $\mathrm{ET}_{\text {sevo }}$ group (group L) and high $\mathrm{ET}_{\text {sevo }}$ group (group $\mathrm{H}$ ).

Peripheral venous blood was collected from all the patients before the induction of general anesthesia (before) and $2 \mathrm{~h}$ after the beginning of surgery (after). The blood samples were then centrifuged for $10 \mathrm{~min}$ at $3000 \mathrm{rpm}$ at $4{ }^{\circ} \mathrm{C}$, and the supernatant plasma was collected and frozen at $-80^{\circ} \mathrm{C}$. After identifying the patients in group $\mathrm{L}$ and $\mathrm{H}$, their serum samples were retrieved and further divided into four groups based on $\mathrm{ET}_{\text {sevo }}$ grouping and sampling time as: L-before, L-after, $\mathrm{H}$-before and $\mathrm{H}$-after groups.

\section{Data processing and multivariate statistical analysis}

In total, $100 \mu \mathrm{L}$ of plasma and $300 \mu \mathrm{L}$ of methanol were mixed. The mixture sonicated for $10 \mathrm{~min}$ and incubated for $1 \mathrm{~h}$ at $-20^{\circ} \mathrm{C}$. Then, the samples were centrifuged at $12,000 \mathrm{rpm}$ for $15 \mathrm{~min}$. The resulting supernatants were transferred to LC-MS vials and stored at $-80^{\circ} \mathrm{C}$. Quality control (QC) samples were prepared by mixing an equal aliquot of the supernatants from all of the samples. LCMS/MS analysis was performed using an UHPLC system (1290, Agilent Technologies) with a UPLC HSS T3 column $(2.1 \mathrm{~mm} \times 100 \mathrm{~mm}, 1.8 \mu \mathrm{m})$ coupled to Q Exactive (Orbitrap MS, Thermo). Mobile phase A was 0.1\% formic acid in water for the positive ion mode and 5 $\mathrm{mmol} \cdot \mathrm{L}^{-1}$ ammonium acetate in water for the negative ion mode, and mobile phase $\mathrm{B}$ was acetonitrile. The QE mass spectrometer was used to acquire MS/MS spectra on an information-dependent basis during the LC/MS experiment. In this mode, the acquisition software (Xcalibur 4.0.27, Thermo) continuously evaluated the fullscan survey MS data. MS raw data from the UHPLC system were converted to the mzML format using ProteoWizard. Then, the data were filtered using the followed criterion: less than $50 \%$ all sample numbers in a group contained a metabolite (QC samples were also taken as a group). Next, missing values were replaced by half of the minimum value in the data set by default [14]. OSI-SMMS (Version 1.0, Dalian Chem Data Solution Information Technology Co. Ltd.) was used for the self-built database after XCMS (Version 3.2) data processing. The repeated metabolites from the positive and negative ion modes were merged.

Orthogonal projection to latent structures-discriminant analysis (OPLS-DA) was applied for between-group comparisons using $\mathrm{R}$ package models. The OPLS-DA model was further validated by cross-validation and permutation test. The predicted parameters of OPLS-DA model included $R^{2} X, R^{2} Y$ and predictive ability $\left(Q^{2}\right)$ values. $R^{2} X$ and $R^{2} Y$ represent the interpretation rate of the model to $\mathrm{X}$ and $\mathrm{Y}$ matrices. $\mathrm{Q}^{2}$ values represented the most recognized diagnostic statistical parameter to validate the
OPLS-DA model in metabolomics. Acceptable predictive model is considered for $\mathrm{Q}^{2}$ value greater than 0.5 [15].

\section{Differential metabolites and pathway analysis}

The variable importance in projection (VIP) score of the OPLS-DA model was applied to rank the metabolites that best distinguished between comparisons. A $t$-test was also used for univariate analysis to screen differential metabolites. Metabolites with $P<0.05$ and VIP $\geq 1$ were considered differential metabolites between comparisons [16]. Metabolites were mapped to KEGG [17] metabolic pathways for pathway and enrichment analyses. $P<0.05$ was statistical significance. Pathways meeting this condition were defined as significantly enriched pathways for differential metabolites.

\section{Statistical analysis}

Statistical analyses were performed using SPSS (Version 22.0, IBM Corp., Armonk, NY, USA). Normally distributed data were presented as the mean $\pm \mathrm{SD}$, whereas non-normally distributed data were presented as the median and interquartile range. Analysis of variance was used for continuous variables. Categorical data were presented as numbers and compared using the Chi-squared test. Groups were compared using Student's $t$-test (normally distributed data) or the Mann-Whitney $U$ test (skewed data). To identify correlations between metabolite levels and the amount of sevoflurane exposure, stepwise multivariate linear regression was used, and Pearson's correlation analysis was applied using MetaboAnalyst 4.0.

\section{Results}

\section{Characteristics of patients}

In total, 500 patients were enrolled, and no patient reported intraoperative recall. The population mean $\mathrm{ET}_{\text {sevo }}$ of the 500 patients was $1.60 \% \pm 0.34 \%$. Seventytwo patients were low $\mathrm{ET}_{\text {sevo }}\left(\mathrm{ET}_{\text {sevo }}<1.26 \%\right)$ (group L), and 102 patients were high $\mathrm{ET}_{\text {sevo }}\left(\mathrm{ET}_{\text {sevo }}>1.94 \%\right)$ (group $\mathrm{H}$ ). According to the principle of pairing, we paired 55 patients from the group $\mathrm{L}$ and 59 patients from the group $\mathrm{H}$. The mean $\mathrm{ET}_{\text {sevo }}$ of the group $\mathrm{L}$ was $1.06 \% \pm 0.13 \%$, which was significantly lower than that of group $\mathrm{H}(2.17 \% \pm 0.16 \%, P<0.001)$. All other primary characteristics of the patients were similar between the two groups (supplementary Table 1). The flow chart of the trial scheme is shown in supplementary Fig. 1.

\section{Multivariate statistical analysis}

Two OPLS-DA models were built using a dataset including the four group samples, with validation parameters as follows: $\mathrm{Q}^{2}=0.592$ (L-before vs. L-after, supplementary Table 2); $\mathrm{Q}^{2}=0.667$ (H-before vs. $\mathrm{H}$-after, supplementary Table 2). The score plots revealed that each class was well 
separated, suggesting that the OPLS-DA model successfully discriminated samples according to their underlying metabolic profiles (Fig. 1a, b). However, the model data did not predict distinction at the same time point between groups as L-before vs. H-before and L-after vs. $\mathrm{H}$-after $\left(Q^{2}<0.5\right)$ (supplementary Table 2$)$. The distribution of sample dots between the two groups for L-before vs. Hbefore and L-after vs. $\mathrm{H}$-after overlapped well, suggesting the levels of most metabolites were similar at the same time point between the two groups (Fig. 1c, d).

\section{Identification of potential regulated metabolites}

For all selected patients, 2356 metabolites were significantly changed between before the induction of anesthesia and $2 \mathrm{~h}$ after the beginning of surgery, while 295 metabolites were detected after filters. In group L, 1558 metabolites were significantly changed between before the induction of anesthesia and $2 \mathrm{~h}$ after the beginning of surgery, while 93 metabolites were detected after filters. In group H, 1553 metabolites were significantly changed between before the induction of anesthesia and $2 \mathrm{~h}$ after the beginning of surgery and 91 metabolites were detected after filters. Compared to group H, 1562 metabolites were significantly changed before the induction of anesthesia in group L, but only 25 metabolites were identified after filters. Compared to group H, 1562 metabolites were significantly changed at $2 \mathrm{~h}$ after the beginning of surgery in group L, but only 38 metabolites were identified after
A

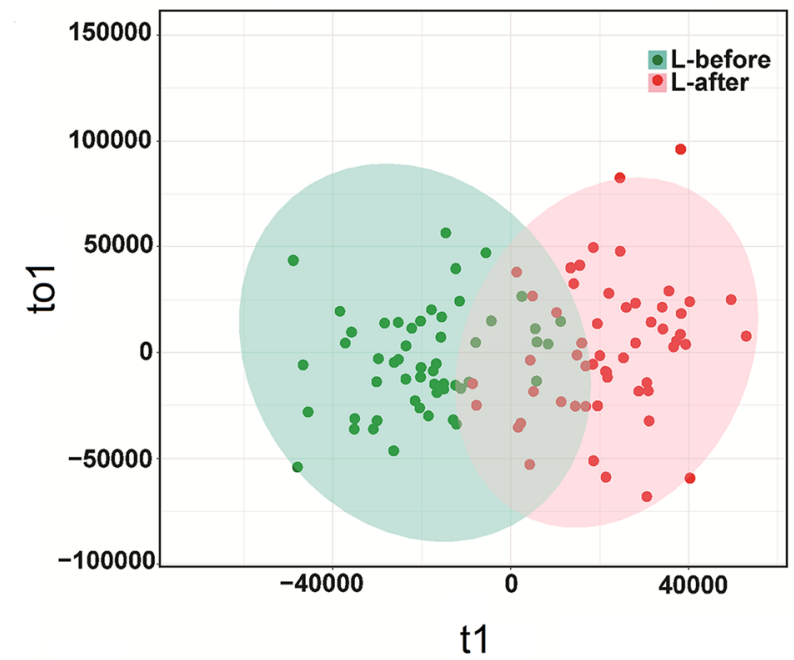

C

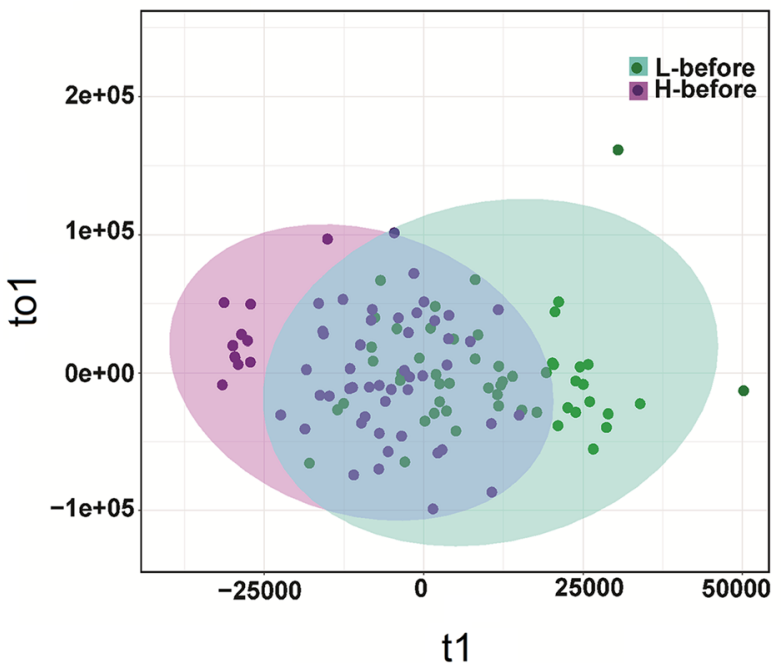

B

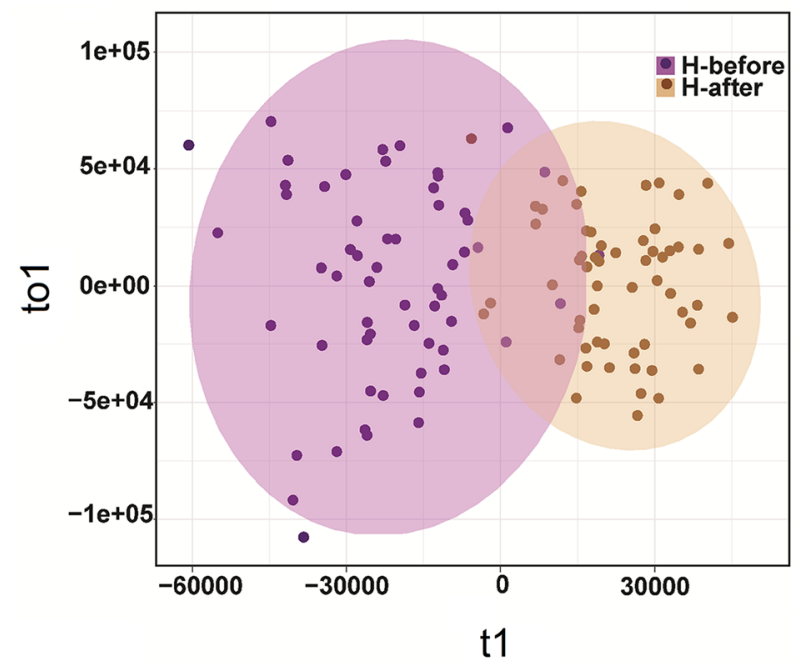

D

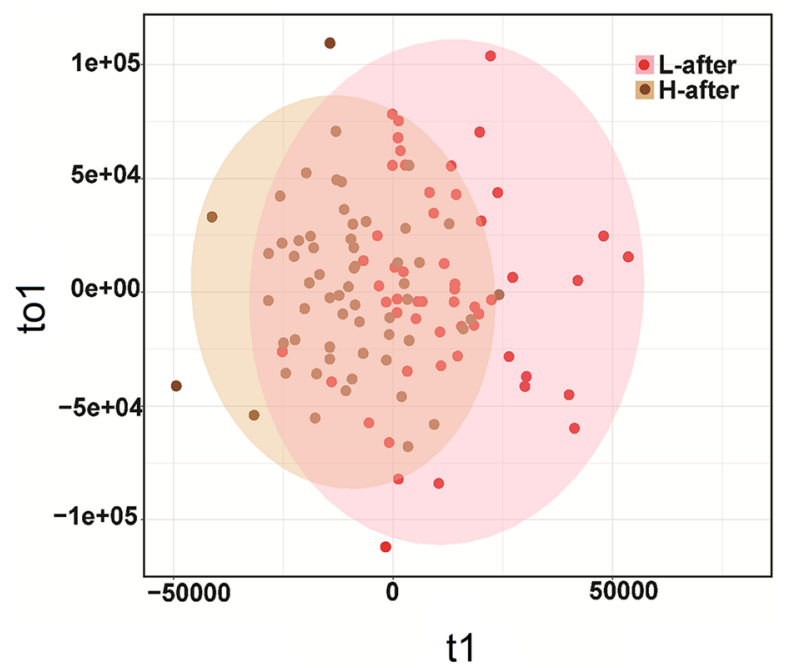

Fig. 1 OPLS-DA score plots between the L-before and L-after groups and between the H-before and H-after groups (a, b). OPLS-DA score plots between the L-before and L-after groups and between the H-before and $\mathrm{H}$-after groups $(\mathbf{c}, \mathbf{d})$ 
filters. OPLS-DA was used to screen differential metabolites. Metabolites with $P<0.05$ and VIP $\geq 1$ were considered differential metabolites between comparisons [16]. For all selected patients, the levels of seventeen metabolites were significantly increased after anesthesia/abdominal surgery, while thirteen metabolites decreased (Table 1). According to the changed metabolites after anesthesia/abdominal surgery, sevoflurane anesthesia and abdominal surgery disturbed the tricarboxylic acid (TCA) cycle as identified by increased citrate and cis-aconitate levels, and impacted glycometabolism as identified by increased sucrose and D-glucose levels in all selected patients. Sevoflurane anesthesia and abdominal surgery also impacted the levels of other metabolites such as phenylethylamine, L-carnitine and nicotine.

The significantly changed metabolites within the subgroups (group L or group $\mathrm{H}$ ) before and after anesthesia/abdominal surgery were also analysed and presented as heatmaps in Fig. 2. In the group L, the levels of five metabolites were significantly increased after anesthesia/abdominal surgery, whereas ten metabolites decreased (supplementary Table 3 ). In the group $\mathrm{H}$, the levels of ten metabolites were significantly increased after anesthesia/abdominal surgery, while thirteen metabolites decreased (supplementary Table 4).

Table 1 Up- and down-regulated metabolites between before anesthesia and $2 \mathrm{~h}$ after starting surgery in all patients

\begin{tabular}{|c|c|c|c|c|}
\hline Metabolites & Fold change & $P$-value & q-value & VIP \\
\hline N-Acetyl-D-glucosamine 6-phosphate & 6.581917 & $2.67 \mathrm{E}-14$ & $4.98 \mathrm{E}-13$ & 2.902062 \\
\hline AMP & 4.707389 & $2.42 \mathrm{E}-13$ & $3.68 \mathrm{E}-12$ & 1.145699 \\
\hline Ephedrine & 1.888023 & 0.000189 & 0.000568 & 1.898164 \\
\hline PC (15:0/20:0) & 1.775193 & 0.001708 & 0.004864 & 1.232962 \\
\hline Sucrose & 1.712725 & 3.36E-05 & 0.000114 & 1.409021 \\
\hline 4-Ketocyclophosphamide & 1.494188 & 0.007404 & 0.015887 & 2.021637 \\
\hline Acyclovir & 1.402124 & 4.21E-07 & $1.92 \mathrm{E}-06$ & 1.021479 \\
\hline Se-Methyl-L-selenocysteine & 0.826514 & 0.000576 & 0.001884 & 2.999008 \\
\hline D-erythro-1-(Imidazol-4-yl) glycerol-3-phosphate & 0.73012 & $1.66 \mathrm{E}-10$ & $1.92 \mathrm{E}-09$ & 1.236423 \\
\hline Citrate & 0.381647 & $8.53 \mathrm{E}-07$ & 4.95E-06 & 4.650407 \\
\hline 4,6-Dichloro-3-methylcatechol & 0.361359 & 4.00E-06 & $2.06 \mathrm{E}-05$ & 1.123057 \\
\hline L-ribulose & 0.333307 & $4.88 \mathrm{E}-12$ & $5.66 \mathrm{E}-11$ & 1.20026 \\
\hline cis-Aconitic acid & 0.316411 & $1.42 \mathrm{E}-05$ & $6.69 \mathrm{E}-05$ & 1.128694 \\
\hline D-glucose & 0.303546 & $5.18 \mathrm{E}-12$ & $5.95 \mathrm{E}-11$ & 6.04479 \\
\hline L-glutamine & 0.225095 & 2.67E-10 & $2.44 \mathrm{E}-09$ & 1.856811 \\
\hline L-selenocysteine & 0.210814 & $1.33 \mathrm{E}-08$ & 7.61E-08 & 2.994583 \\
\hline Pyroglutamic acid & 0.150226 & $7.92 \mathrm{E}-05$ & 0.063248 & 1.814755 \\
\hline Se-Methylselenomethionine & -5.14371 & $1.02 \mathrm{E}-18$ & 3.19E-07 & 1.97583 \\
\hline Phenylethylamine & -1.99326 & 4.77E-26 & 5.97E-24 & 2.200773 \\
\hline Glycochenodeoxycholic acid & -1.42167 & 0.000184 & 0.000555 & 1.278177 \\
\hline 5-Acetylamino-6-formylamino-3-methyluracil & -0.84274 & 4.91E-14 & 7.87E-13 & 1.313026 \\
\hline Cholesterol sulfate & -0.59075 & $9.72 \mathrm{E}-08$ & $6.45 \mathrm{E}-07$ & 1.349612 \\
\hline Bilirubin & -0.37302 & 0.000103 & 0.000417 & 1.323645 \\
\hline LysoPC (18:2(9Z,12Z)) & -0.36135 & 8.93E-11 & 7.23E-10 & 12.17143 \\
\hline dTDP-3-methyl-4-oxo-2,6-dideoxy-L-allose & -0.31602 & $2.38 \mathrm{E}-07$ & $1.13 \mathrm{E}-06$ & 3.282894 \\
\hline Nicotine & -0.28852 & $1.49 \mathrm{E}-10$ & 1.17E-09 & 3.286386 \\
\hline L-carnitine & -0.28411 & $1.25 \mathrm{E}-10$ & $9.95 \mathrm{E}-10$ & 11.95738 \\
\hline 2-Oxoglutaramate & -0.25893 & 4.79E-05 & 0.000167 & 1.722153 \\
\hline Sphinganine & -0.23381 & $3.38 \mathrm{E}-10$ & $2.54 \mathrm{E}-09$ & 1.935018 \\
\hline 4'-O-Demethylrebeccamyc & -0.16481 & 0.002641 & 0.007141 & 2.88872 \\
\hline
\end{tabular}

VIP Variable importance in the projection. Positive values in fold change indicate increases while negative values indicate decreases. $\mathrm{q}$-value: False discovery rate adjusted $p$-values 


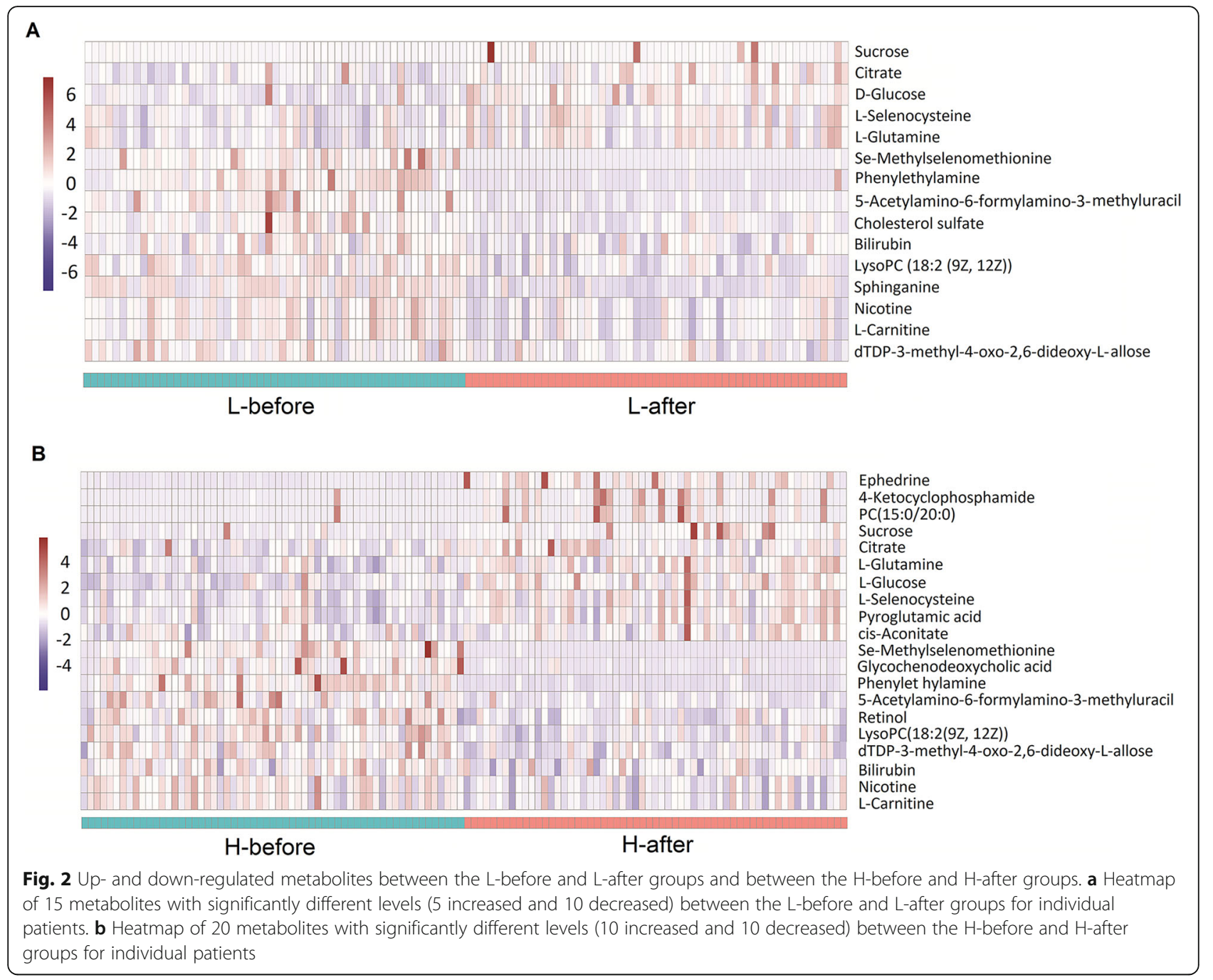

The significantly changed metabolites at the same time point between group $\mathrm{L}$ and $\mathrm{H}$ were presented as heatmaps in supplementary Fig. 2. Before anesthesia, compared with group L, only one metabolite was higher and two metabolites were lower in group $\mathrm{H}$ (supplementary Table 5). After anesthesia/abdominal surgery, the levels of seven metabolites were higher in group $\mathrm{H}$ than those in group L, whereas three metabolites were lower in group $\mathrm{H}$ (supplementary Table 6).

Theoretically, changed metabolites of all selected patients (Table 1) or common changed metabolites of group $\mathrm{H}$ and group $\mathrm{L}$ after anesthesia/abdominal surgery mainly represent the metabolic pathways impacted by anesthesia/abdominal surgery (Fig. 3a). While the differentially changed metabolites after anesthesia/abdominal surgery between group $\mathrm{H}$ and group $\mathrm{L}$ may represent the metabolic pathways relevant to the amount of sevoflurane exposure or sevoflurane sensitivity (Fig. 3b). Our results showed that the levels of 5-aminopentanoate, L-glutamine, pyroglutamic acid, L-selenocysteine and sphinganine in group L after anesthesia/abdominal surgery were higher than those in group $\mathrm{H}$.

\section{Biological pathway analysis}

Pathway analysis was applied to investigate biological functions of the altered metabolites. Multiple metabolic pathways were impacted after anesthesia/abdominal surgery in both group L (supplementary Fig. 3A) and group $\mathrm{H}$ (supplementary Fig. 3B), mainly including carbohydrate digestion and absorption, ABC transporters, mineral absorption, glutamate metabolism. Interestingly, some metabolic pathways were only impacted in $\mathrm{H}$ group (supplementary Fig. 3B) such as glutamatergic synapse and GABAergic synapse, which might account for higher requirement of sevoflurane for these patients or higher amount of sevoflurane exposure. The involvement of the altered metabolites in KEGG pathways between $\mathrm{L}$ and $\mathrm{H}$ groups at the same time (before or after anesthesia/abdominal surgery) was shown in supplementary Fig. 4. 

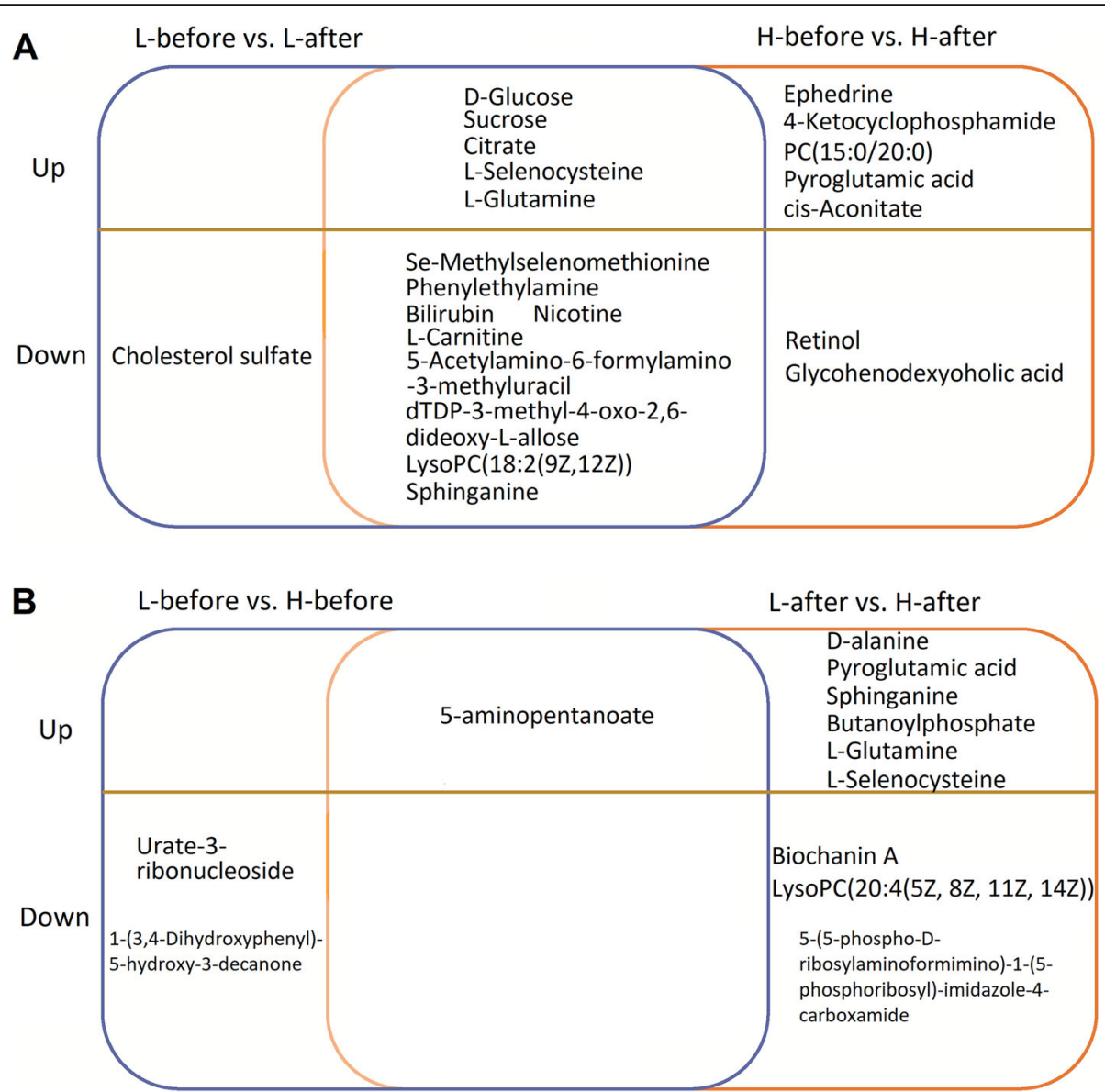

Fig. 3 Up- and down-regulated metabolites among the L-before, L-after, $\mathrm{H}$-before and $\mathrm{H}$-after groups. a Up- and down-regulated metabolites between the L-before and L-after groups and between the H-before and H-after groups. b Up- and down-regulated metabolites between the Lbefore and $\mathrm{H}$-before groups and between the L-after and $\mathrm{H}$-after groups

\section{Changes of four metabolites after anesthesia/abdominal} surgery are relevant to the amount of sevoflurane exposure Glutamate metabolism might be critical for sevoflurane anesthesia and sensitivity. L-glutamine, L-selenocysteine, pyroglutamic acid involve in glutamate metabolism. Sevoflurane anesthesia and abdominal surgery increased L-glutamine, pyroglutamic acid, L-glutamine, pyroglutamic acid and 5-aminopentanoate levels in the group $\mathrm{H}$ compared with that those in the group L. Sevoflurane anesthesia and abdominal surgery decreased sphinganine level, and its level was higher in the $\mathrm{H}$ group after anesthesia. Correlation analysis identified four metabolites that may be relevant to the sevoflurane requirement (Area under curve of $\mathrm{ET}_{\text {sevo }}$, Fig. 4a): L-glutamine (Fig. 4b, $\mathrm{P}=0.0435$ ), L-selenocysteine (Fig. 4c, $\mathrm{P}=$ 0.0137), pyroglutamic acid (Fig. $4 \mathrm{~d}, \mathrm{P}=0.0008$ ) and sphinganine (Fig. 4e, $\mathrm{P}=0.0015$ ). These higher increased metabolites in the group $\mathrm{H}$ after anesthesia/abdominal surgery may result from higher amount of sevoflurane exposure. In turn, such differential metabolic status might determine sevoflurane requirement.

\section{Discussion}

The present study indicated that sevoflurane anesthesia and abdominal surgery can significantly impact major metabolic pathways in human. Sevoflurane and abdominal surgery increased citrate, cis-aconitate, sucrose and D-glucose levels in patients. Glutamate metabolism was also impacted by sevoflurane and abdominal surgery. In the patients with high ETsevo, levels of L-glutamine, pyroglutamic acid, sphinganine and L-selenocysteine after sevoflurane anesthesia and abdominal surgery were significantly higher than those of the patients with low ETsevo, suggesting that these metabolic changes might be relevant to the amount of sevoflurane exposure.

Sevoflurane anesthesia has been known to impact the systemic metabolism in animals. For example, sevoflurane anesthesia increases the blood concentration of glucose and decreases blood concentration of lactate in rodents [18]. Lipid metabolism is also disturbed after sevoflurane anesthesia in monkeys, for example, lipid species including polyunsaturated fatty acids are depleted after sevoflurane anesthesia [19]. However, how sevoflurane 
A

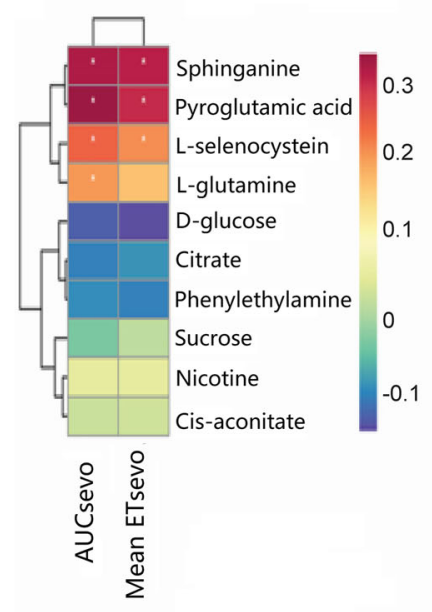

B

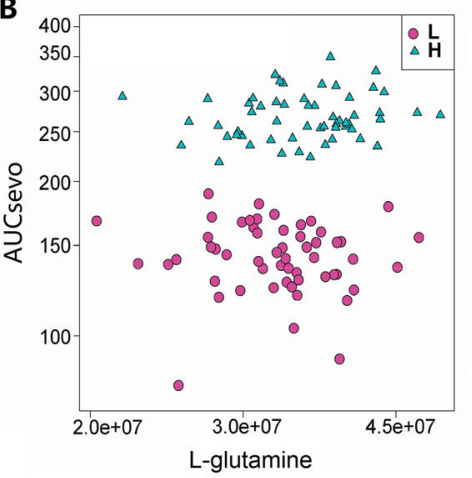

D

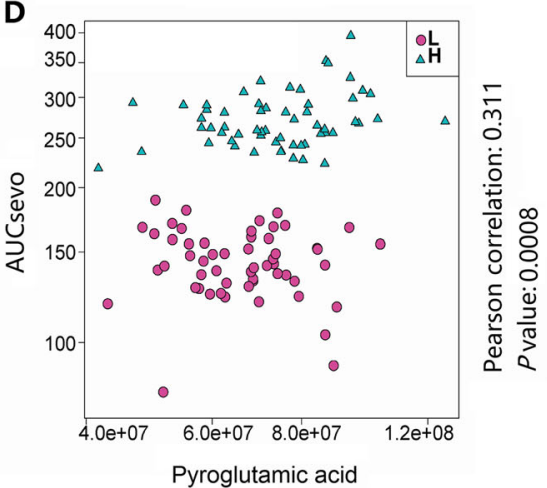

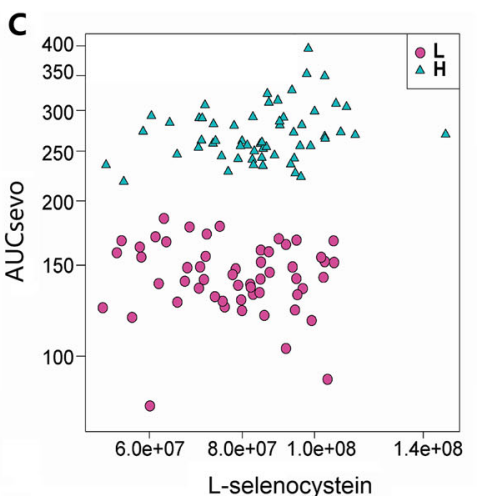

C

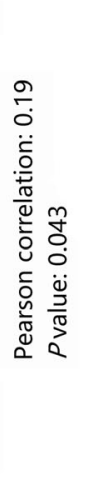

E

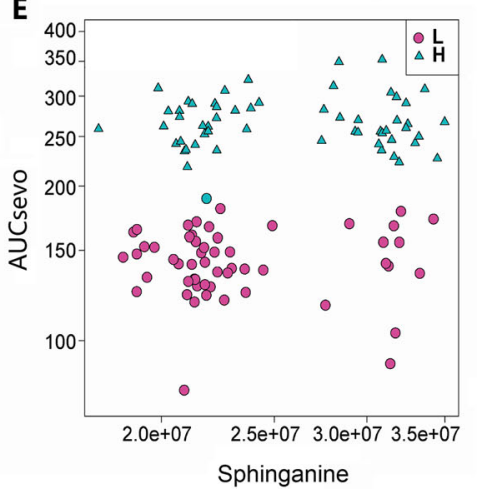

Fig. 4 Correlation analysis between metabolites and AUC-ET $T_{\text {sevo. }}$. a Correlation analysis between 10 metabolites and AUC-ET sevo. Four metabolites, including L-glutamine (b), L-selenocysteine (c), pyroglutamic acid (d) and sphinganine (e) were correlated with AUC-ET sevo

anesthesia and surgery impact systemic metabolomics in clinical patients is largely unknown.

In this study, we compared alteration of systemic metabonomics after sevoflurane anesthesia and abdominal surgery in human subjects. Our results indicate that sevoflurane anesthesia and abdominal surgery can impact principal metabolic pathways, including the TCA cycle, glycometabolism and glutamate metabolism. The TCA cycle is the final common oxidative pathway connecting all individual metabolic pathways [20]. In this cycle, citrate is converted to isocitrate via cis-aconitate by aconitase [20], and citrate can be exported from the mitochondria through citrate carriers [20]. Citrate links many important cellular processes, bridging carbohydrate and fatty acid metabolism and protein modification. The results here indicated that citrate and cis-aconitate were accumulated after sevoflurane anesthesia, suggesting that the TCA cycle might be impeded during general anesthesia.

Previous studies of animals indicate that sevoflurane may disrupt glucose metabolism [2], insulin secretion [21], insulin resistance and glucose uptake [2, 6]. Elevated glucose level is associated with increased infection rates after general surgeries [22]. The expression of several pro-inflammatory cytokines is regulated by glucose levels, including tumour necrosis factor TNF- $\alpha$ [23] and interleukin IL-1 $\beta$ [10]. The impairment of neutrophil degranulation induced by hyperglycaemia is also relevant to infection after surgery [10]. Our results indicated that sevoflurane anesthesia and abdominal surgery increased sucrose and D-glucose levels in both groups, which might increase the infection risks of postoperative patients.

Alteration of metabonomics after anesthesia and surgery may also contribute to postoperative cognitive dysfunction (POCD) [24]. Previous study indicates that injection of nicotine after anesthesia induction can markedly prevent POCD in rats [25]. L-carnitine can exert neuroprotective effects via attenuating inflammation and oxidative damage [26]. Low phenylethylamine level in children is relevant to attention deficit-hyperactivity disorder [27]. The concentration of phenylethylamine has been proposed as a useful biochemical marker in psychiatric and behavioural research [28]. Our results showed that the content of nicotine, Lcarnitine and phenylethylamine in plasma decreased after anesthesia/abdominal surgery compared with those before anesthesia in both groups, which may impact postoperative cognitive functions of patients. 
Interestingly, correlation analysis identified four metabolites potentially relevant to the amount of sevoflurane exposure: L-glutamine, pyroglutamic acid, sphinganine and L-selenocysteine. L-glutamine is involved in glutamate metabolism and glutamate is an essential metabolic precursor in glucose biosynthesis, protein synthesis and glutathione homeostasis [29]. Glutamate is the most abundant excitatory neurotransmitter in the brain and plays a fundamental role in learning and memory [30]. Glutamate released by pre-synaptic neurons is rapidly converted to glutamine in astrocytes and glutamine released from astrocytes can also be converted back to glutamate. The glutamate/glutamine cycle is essential for normal function of synaptic transmission [30]. Depression of excitatory synaptic transmission is considered the principal mechanism of general anesthesia [31]. The level of brain glutamine and glutamate were also altered in dogs when anesthetized with sevoflurane and isoflurane [3]. Volatile anesthetics may increase glutamate uptake by astrocytes, thereby affecting excitatory synaptic transmission in the central nervous system [32]. Our results indicated that the level of L-glutamine was higher in the group $\mathrm{H}$ after anesthesia/abdominal surgery than that in the group L, which might be related to the differential functions in excitatory synaptic transmission and excitatory neurotransmitter release during sevoflurane anesthesia. This may also result from the different requirement of sevoflurane between the group $\mathrm{L}$ and group $\mathrm{H}$.

Pyroglutamic acid, derived from L-glutamic acid in the $\gamma$ glutamyl cycle, is one of the essential components for amino acid transport [33]. Pyroglutamic acid induces antidiabetic properties in vivo and inhibits energy production of the cerebral cortex of rats [34]. Selenocysteine is involved in glutamate metabolism, DNA damage and can inhibit cell growth and mitochondrial function by triggering reactive oxygen species [35]. The inhibition of mitochondrial function is one of the most important mechanisms by which volatile anesthetics exert their anesthetic effect [36]. Our results indicated that the content of pyroglutamic acid and Lselenocysteine in the group $\mathrm{H}$ after anesthesia/abdominal surgery were higher than those in the group L, which may be related to the differential glutamate metabolism during sevoflurane anesthesia.

Sphinganine, an important structural element of cell membranes [37], plays a key role in the biosynthesis and metabolism of sphingolipids [38]. Altered sphingolipid metabolism is potentially related to the inflammatory response and increased ceramide levels [38]. Studies has shown that the reduction of sphinganine in cortical neurons can impact mitochondrial respiratory function [37]. However, the effects of anesthesia/surgery on sphinganine level has not been reported. Our results indicate that the level of sphinganine in the group $\mathrm{H}$ after anesthesia and abdominal surgery were higher than that in the group L.
The present study has several limitations. Firstly, we cannot separately investigate the effect of sevoflurane or abdominal surgery on metabonomics in patients. Therefore, the systemic effects on metabonomics here are the overall effects. Secondly, although the central nervous system is the main target for general anesthetics, we cannot measure the change of metabonomics in the brain.

\section{Conclusions}

In summary, the present study indicated that sevoflurane anesthesia and abdominal surgery can impact principal metabolic pathways in clinical patients. The plasma concentrations of L-glutamine, pyroglutamic acid, sphinganine and L-selenocysteine might be relevant to the amount of sevoflurane exposure. This study may provide a resource data for future studies about metabolism relevant to general anaesthesia and surgeries.

\section{Abbreviations \\ $\mathrm{ET}_{\text {sevo: }}$ : End-tidal sevoflurane concentration; BIS: Bispectral index; $\mathrm{SPO}_{2}$ : Pulse oxygen saturation; BP: Blood pressure; ECG: Electrocardiograph; BIS: Bispectral index; $\mathrm{P}_{\mathrm{ET}} \mathrm{CO}_{2}$ : End-tidal partial pressure of carbon dioxide; $\mathrm{BMI}$ : Body mass index; QC: Quality control; OPLS-DA: Orthogonal projection to latent structures-discriminant analysis; VIP: Variable importance in projection; TCA: Tricarboxylic acid; POCD: Postoperative cognitive dysfunction; AUC: Area under curve}

\section{Supplementary Information}

The online version contains supplementary material available at https://doi. org/10.1186/s12871-021-01301-0.

Additional file 1: Supplementary Table 1. Characteristics of the study patients. Supplementary Table 2. Permutation test results of the OPLSDA models. Supplementary Table 3. Top up- and down-regulated metabolites in the group $L$ before and after sevoflurane anesthesia/surgery. Supplementary Table 4. Top up- and down-regulated metabolites in the group $\mathrm{H}$ before and after sevoflurane anesthesia/surgery. Supplementary Table 5. Up- and down-regulated metabolites between Lbefore group and H-before group. Supplementary Table 6. Up- and down-regulated metabolites between L-after group and $\mathrm{H}$-after group after sevoflurane anesthesia and surgery. Supplementary Figure 1. Flow diagram of the clinical trial. Supplementary Figure 2. Up- and down-regulated metabolites between the L vs. H groups. (A) Heatmap of 12 metabolites with significantly different levels between the L-before and $\mathrm{H}$-before groups. (B) Heatmap of 15 metabolites with significantly different levels between the $\mathrm{L}$-after and $\mathrm{H}$-after groups. Supplementary

Figure 3. Bubble plot of pathway analysis. (A) The pathways that differed between the L-before and L-after groups. (B) The pathways that differed between the $\mathrm{H}$-before and $\mathrm{H}$-after groups. Each dot represents a related metabolic pathway. The colour and size of each dot denote the $-\ln (p)$ value and pathway impact value, respectively. Supplementary Figure 4. Bubble plot of pathway analysis. (A) The pathways that significantly differed between the L-before and H-before groups. (B) The pathways that significantly differed between the $\mathrm{L}$-after and $\mathrm{H}$-after groups. Each dot represents a related metabolic pathway. The colour and size of each dot denote the- $\ln (p)$ value and pathway impact value, respectively.

\section{Acknowledgements}

Not applicable.

Authors' contributions

Study design: YW, DZ, JL, YZ. Study conduct: YW, DZ, MO, PL, CZ. Data analysis: YW, DZ, MO, CZ, YZ. Drafting manuscript: $Y W, D Z, J L, Y Z$. Revising 
manuscript: all authors. The author(s) read and approved the final manuscript.

\section{Funding}

This work was supported by the grants 81571353 (to J.L.) and 81771486 (to C.Z.) from the National Natural Science Foundation of China (NSFC, Beijing, China), and ZY2016101 (to J.L.) from the 1, 3, 5 Project of West China Hospital.

\section{Availability of data and materials}

The datasets used and/or analysed during the current study are available from the corresponding author on reasonable request.

\section{Declarations}

\section{Ethics approval and consent to participate}

This observational study was approved by the Ethical Committee of West China Hospital of Sichuan University (Ref: 2017078) on May 19, 2017, and adhered to the Strengthening the Reporting of Observational studies in Epidemiology (STROBE) guidelines. Written informed consent was obtained from all patients before enrolment.

\section{Consent for publication}

Not applicable.

\section{Competing interests}

The authors declare that they have no competing interests.

Received: 29 September 2020 Accepted: 8 March 2021

Published online: 17 March 2021

\section{References}

1. Du WJ, Zhang RW, Li J, Zhang BB, Peng XL, Cao S, Yuan J, Yuan CD, Yu T, Du JL. The Locus Coeruleus Modulates Intravenous General Anesthesia of Zebrafish via a Cooperative Mechanism. Cell Rep. 2018; 24(12):3146-55 e3143.

2. Høyer KF, Nielsen TS, Risis S, Treebak JT, Jessen N. Sevoflurane impairs insulin secretion and tissue-specific glucose uptake in vivo. Basic Clin Pharmacol Toxicol. 2018;123(6):732-8. https://doi.org/10.1111/bcpt.13087.

3. Sobbeler FJ, Carrera I, Pasloske K, Ranasinghe MG, Kircher P, Kastner SBR. Effects of isoflurane, sevoflurane, propofol and alfaxalone on brain metabolism in dogs assessed by proton magnetic resonance spectroscopy ((1) H MRS). BMC Vet Res. 2018;14(1):69. https://doi.org/10.1186/s12917-01 8-1396-1.

4. Alkire MT, Hudetz AG, Tononi G. Consciousness and anesthesia. Science. 2008;322(5903):876-80.

5. Palanca BJA, Avidan MS, Mashour GA. Human neural correlates of sevoflurane-induced unconsciousness. Br J Anaesth. 2017;119(4):573-82. https://doi.org/10.1093/bja/aex244.

6. Li X, Kitamura T, Kawamura G, Mori Y, Sato K, Araki Y, Sato R, Yamada Y. Comparison of mechanisms underlying changes in glucose utilization in fasted rats anesthetized with propofol or sevoflurane: Hyperinsulinemia is exaggerated by propofol with concomitant insulin resistance induced by an acute lipid load. Biosci Trends. 2014;8(3):155-62. https://doi.org/10.5582/ bst.2014.01060.

7. Kim H, Han J. Comparison of sevoflurane and propofol anesthesia on the incidence of hyperglycemia in patients with type 2 diabetes undergoing lung surgery. Yeungnam Univ J Med. 2018;35(1):54-62.

8. Lozano AJ, Brodbelt DC, Borer KE, Armitage-Chan E, Clarke KW, Alibhai HI. A comparison of the duration and quality of recovery from isoflurane, sevoflurane and desflurane anaesthesia in dogs undergoing magnetic resonance imaging. Vet Anaesth Analg. 2009;36(3):220-9. https://doi.org/1 0.1111/j.1467-2995.2009.00451.x.

9. Kim M, Snowden S, Suvitaival T, Ali A, Merkler DJ, Ahmad T, Westwood S, Baird A, Proitsi P, Nevado-Holgado A, Hye A, Bos I, Vos S, Vandenberghe R, Teunissen C, ten Kate M, Scheltens P, Gabel S, Meersmans K, Blin O, Richardson J, de Roeck E, Sleegers K, Bordet R, Rami L, Kettunen P, Tsolaki M, Verhey F, Sala I, Lléo A, Peyratout G, Tainta M, Johannsen P, Freund-Lev Y, Frölich L, Dobricic V, Engelborghs S, Frisoni GB, Molinuevo JL, Wallin A, Popp J, Martinez-Lage P, Bertram L, Barkhof F, Ashton N, Blennow K, Zetterberg H, Streffer J, Visser PJ, Lovestone S, Legido-Quigley C. Primary fatty amides in plasma associated with brain amyloid burden, hippocampal volume, and memory in the European medical information framework for Alzheimer's disease biomarker discovery cohort. Alzheimers Dement. 2019; 15(6):817-27. https://doi.org/10.1016/j.jalz.2019.03.004.

10. Bux AS, Lindsey ML. Glucose regulates the intrinsic inflammatory response of the heart to surgically induced hypothermic ischemic arrest and reperfusion. Physiol Genomics. 2017;49(1):37-52.

11. Zimin PI, Woods CB, Kayser EB, Ramirez JM, Morgan PG, Sedensky MM. Isoflurane disrupts excitatory neurotransmitter dynamics via inhibition of mitochondrial complex I. Br J Anaesth. 2018;120(5):1019-32. https://doi. org/10.1016/j.bja.2018.01.036.

12. Ramadasan-Nair R, Hui J, Zimin PI, Itsara LS, Morgan PG. Regional knockdown of NDUFS4 implicates a thalamocortical circuit mediating anesthetic sensitivity. PloS One. 2017;12(11):e0188087.

13. Falk MJ, Kayser EB, Morgan PG, Sedensky MM. Mitochondrial complex I function modulates volatile anesthetic sensitivity in C. elegans. Curr Biol. 2006;16(16):1641-5. https://doi.org/10.1016/j.cub.2006.06.072.

14. Xia J, Psychogios N, Young N, Wishart DS. MetaboAnalyst: a web server for metabolomic data analysis and interpretation. Nucl Acids Res. 2009;37(Web Server issue):W652-60.

15. Triba MN, Le Moyec L, Amathieu R, Goossens C, Bouchemal N, Nahon P, Rutledge DN, Savarin P. PLS/OPLS models in metabolomics: the impact of permutation of dataset rows on the K-fold cross-validation quality parameters. Mol BioSyst. 2015;11(1):13-9. https://doi.org/10.1039/C4 MB00414K.

16. Chen C, Luo F, Wu P, Huang Y, Das A, Chen S, Chen J, Hu X, Li F, Fang Z. Metabolomics reveals metabolite changes of patients with pulmonary arterial hypertension in China. J Cell Mole Med. 2020;24(4):2484-96.

17. Kanehisa M, Goto S. KEGG: Kyoto encyclopedia of genes and genomes. Nucleic Acids Res. 2000;28(1):27-30. https://doi.org/10.1093/nar/28.1.27.

18. Du H, Li S, Zhang Y, Guo H, Wu L, Liu H, Manyande A, Xu F, Wang J. NMR Based Metabolomics Comparison of Different Blood Sampling Techniques in Awake and Anesthetized Rats. Molecules. 2019;24(14):2542.

19. Wang C, Liu F, Frisch-Daiello JL, Martin S, Patterson TA, Gu Q, Liu S, Paule MG, Hanig JP, Slikker W Jr, Crawford PA, Wang C, Han X. Lipidomics reveals a systemic energy deficient state that precedes neurotoxicity in neonatal monkeys after sevoflurane exposure. Anal Chim Acta. 2018;1037:87-96. https://doi.org/10.1016/j.aca.2017.11.052.

20. Akram M. Citric acid cycle and role of its intermediates in metabolism. Cell Biochem Biophys. 2014;68(3):475-8. https://doi.org/10.1007/s12013-0139750-1.

21. Tanaka T, Nabatame H, Tanifuji Y. Insulin secretion and glucose utilization are impaired under general anesthesia with sevoflurane as well as isoflurane in a concentration-independent manner. J Anesth. 2005;19(4):277-81. https://doi.org/10.1007/s00540-005-0341-1.

22. Pomposelli JJ, Baxter JK 3rd, Babineau TJ, Pomfret EA, Driscoll DF, Forse RA, Bistrian BR. Early postoperative glucose control predicts nosocomial infection rate in diabetic patients. JPEN J Parenter Enteral Nutr. 1998;22(2): 77-81. https://doi.org/10.1177/014860719802200277.

23. Wu Y, Song LT, Li JS, Zhu DW, Jiang SY, Deng JY. MicroRNA-126 regulates inflammatory cytokine secretion in human gingival fibroblasts under high glucose via targeting tumor necrosis factor receptor associated factor 6 . J Periodontol. 2017:88(11):e179-87. https://doi.org/10.1902/jop.2017.170091.

24. Xiong $L$, Duan $L, X u$ W, Wang Z. Nerve growth factor metabolic dysfunction contributes to sevoflurane-induced cholinergic degeneration and cognitive impairments. Brain Res. 1707;2019:107-16.

25. Wei P, Zheng Q, Liu H, Wan T, Zhou J, Li D, Zhou H, Li J, Ji F, Tang W, Li J. Nicotine-induced Neuroprotection against cognitive dysfunction after partia hepatectomy involves activation of BDNF/TrkB signaling pathway and inhibition of NF-kappaB signaling pathway in aged rats. Nicotine Tob Res. 2018;20(4):515-22. https://doi.org/10.1093/ntr/ntx157.

26. Ferreira GC, McKenna MC. L-Carnitine and acetyl-L-carnitine roles and Neuroprotection in developing brain. Neurochem Res. 2017;42(6):1661-75. https://doi.org/10.1007/s11064-017-2288-7.

27. Kusaga A, Yamashita Y, Koeda T, Hiratani M, Kaneko M, Yamada S, Matsuishi T. Increased urine phenylethylamine after methylphenidate treatment in children with ADHD. Ann Neurol. 2002;52(3):372-4. https://doi.org/10.1002/a na.10302.

28. Giulivi C, Napoli E, Tassone F, Halmai J, Hagerman R. Plasma biomarkers for monitoring brain pathophysiology in FMR1 Premutation carriers. Front Mol Neurosci. 2016;9:71. 
29. Tapiero H, Mathe G, Couvreur P, Tew KD. II. Glutamine and glutamate. Biomed Pharmacother. 2002;56(9):446-57.

30. Lacreuse A, Moore CM, LaClair M, Payne L, King JA. Glutamine/glutamate (Glx) concentration in prefrontal cortex predicts reversal learning performance in the marmoset. Behav Brain Res. 2018;346:11-5. https://doi. org/10.1016/j.bbr.2018.01.025.

31. Hao X, Ou M, Zhang D, Zhao W, Yang Y, Liu J, Yang H, Zhu T, Li Y, Zhou C. The effects of general anesthetics on synaptic transmission. Curr Neuropharmacol. 2020;18(10):936-65. https://doi.org/10.2174/1570159X1 8666200227125854.

32. Miyazaki H, Nakamura Y, Arai T, Kataoka K. Increase of glutamate uptake in astrocytes: a possible mechanism of action of volatile anesthetics. Anesthesiology. 1997;86(6):1359-66 discussion 1358A.

33. Abu Bakar MH, Sarmidi MR. Association of cultured myotubes and fasting plasma metabolite profiles with mitochondrial dysfunction in type 2 diabetes subjects. Mol BioSyst. 2017;13(9):1838-53. https://doi.org/10.1039/ C7MB00333A

34. Sudomova M, Hassan STS. A Multi-Biochemical and In Silico Study on AntiEnzymatic Actions of Pyroglutamic Acid against PDE-5, ACE, and Urease Using Various Analytical Techniques: Unexplored Pharmacological Properties and Cytotoxicity Evaluation. Biomolecules. 2019;9(9):392.

35. Fan CD, Fu XY, Zhang ZY, Cao MZ, Sun JY, Yang MF, Fu XT, Zhao SJ, Shao $L R$, Zhang $H F$, Yang $X Y$, Sun BL. Selenocysteine induces apoptosis in human glioma cells: evidence for TrxR1-targeted inhibition and signaling crosstalk. Sci Rep. 2017;7(1):6465. https://doi.org/10.1038/s41598-017-06979-2.

36. Yamakage M. Can age-related mitochondrial dysfunction affect volatile anesthetic potency? J Anesth. 2014;28(6):805-6. https://doi.org/10.1007/ s00540-014-1907-6.

37. Agostini M, Niklison-Chirou MV, Annicchiarico-Petruzzelli MM, Grelli S, Di Daniele N, Pestlikis I, Knight RA, Melino G, Rufini A. p73 regulates primary cortical neuron metabolism: a global metabolic profile. Mol Neurobiol. 2018. 55(4):3237-50. https://doi.org/10.1007/s12035-017-0517-3.

38. Ghidoni R, Caretti A, Signorelli P. Role of Sphingolipids in the pathobiology of lung inflammation. Mediat Inflamm. 2015;2015:487508.

\section{Publisher's Note}

Springer Nature remains neutral with regard to jurisdictional claims in published maps and institutional affiliations.

Ready to submit your research? Choose BMC and benefit from:

- fast, convenient online submission

- thorough peer review by experienced researchers in your field

- rapid publication on acceptance

- support for research data, including large and complex data types

- gold Open Access which fosters wider collaboration and increased citations

- maximum visibility for your research: over $100 \mathrm{M}$ website views per year

At $\mathrm{BMC}$, research is always in progress.

Learn more biomedcentral.com/submissions 